\title{
ELM triggering conditions for the integrated modeling of H-mode plasmas
}

\author{
A. Y. Pankin ${ }^{\mathrm{a}, 1}$ G. Bateman ${ }^{\text {b }}$ D. P. Brennan ${ }^{\text {c }}$ D. D. Schnack ${ }^{\text {a }}$ P. B. Snyder ${ }^{\mathrm{d}}$

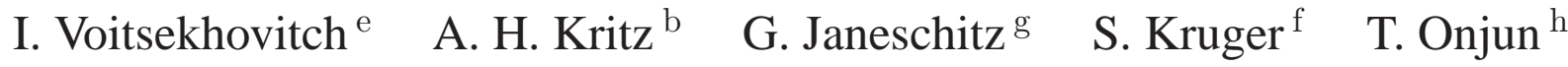 \\ G. W. Pacher ${ }^{\mathrm{i}}$ H. D. Pacher ${ }^{\mathrm{j}}$

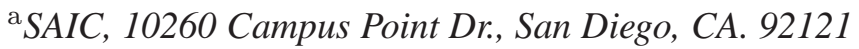 \\ ${ }^{\mathrm{b}}$ Lehigh University, Bethlehem, PA 18015, USA \\ ${ }^{\mathrm{c}}$ MIT, Cambridge, MA 02139 \\ ${ }^{\mathrm{d}}$ General Atomics, San Diego, CA 92186 \\ e JET-UKAEA, Culham Science Centre, UK \\ ${ }^{\mathrm{f}}$ Tech-X, Boulder, $C O \mathrm{O} 80303$ \\ ${ }^{\mathrm{g}}$ FZK-PL-Fusion, Karlsruhe, Germany \\ ${ }^{\mathrm{h}}$ SIIT, Klong Luang, Pathumthani 12121, Thailand \\ ${ }^{\mathrm{i}}$ Hydro-Québec, Varennes, Québec, Canada \\ jINRS, Québec, Canada
}

\begin{abstract}
Recent advances in the integrated modeling of ELMy H-mode plasmas are presented. A model for the $\mathrm{H}$-mode pedestal and for the triggering of ELMs predicts the height, width, and shape of the H-mode pedestal and the frequency and width of ELMs. Formation of the pedestal and the L-H transition is the direct result of $\vec{E}_{r} \times \vec{B}$ flow shear suppression of anomalous transport. The periodic ELM crashes are triggered by either the ballooning or peeling MHD instabilities. The BALOO, DCON, and ELITE ideal MHD stability codes are used to derive a new parametric expression for the peeling-ballooning threshold. The new dependence for the peeling-ballooning threshold is implemented in the ASTRA transport code. Results of integrated modeling of DIII-D like discharges are presented and compared with experimental observations. The results from the ideal MHD stability codes are compared with results from the resistive MHD stability code NIMROD.
\end{abstract}

\section{Introduction}

Transport modeling of the edge of tokamak plasmas is a challenging problem, because a wide range of time and length scales need to be considered and many different elements of physics are involved at the plasma edge. The physics topics that are critically important for the plasma edge are the transition from low- to high- confinement regime (L-H transition), H-mode pedestal build up, anomalous and neoclassical transport at the plasma edge, role of the $\vec{E}_{r} \times \vec{B}$ flow shear, triggering and dynamics of the edge localized modes (ELMs). One of the effective ways to test ideas for physics models is to combine them within an integrative modeling code and compare the simulation results with the experiments. Integrated modeling studies that self-consistently take into account the effects of the plasma edge have been developing recently $[1,2,3]$. In particular, a new

1 E-mail: alexei.y.pankin@ saic.com 
model for the H-mode pedestal and ELMs has been recently developed by Pankin et al [3]. The model predicts the height, width, and shape of the H-mode pedestal as well as the frequency and width of ELMs. The model for the H-mode pedestal in tokamak plasmas is based on flow shear reduction of anomalous transport. The formation of the pedestal and the L-H transition in this model are the direct result of $\vec{E}_{r} \times \vec{B}$ flow shear suppression of transport. ELMs can be triggered either by ballooning or by peeling modes. The model for the pedestal and ELMs has been used in a predictive integrated modeling code to follow the time evolution of tokamak discharges from Lmode through the transition from L-mode to H-mode, with the formation of the H-mode pedestal, and, subsequently, the triggering of ELMs. The model for the H-mode pedestal and ELMs [3] is advanced in this paper. The ELM triggering conditions are studied with the MHD stability codes BALOO [4], DCON [5], and ELITE [6]. These MHD instability codes are used to compute the combined peeling-ballooning threshold, which are then used to derive fitting expressions that are included in the model. Using these MHD instability enhances the model and extends the level of its applicability. The improved stability criterion model is tested in the integrated modeling code ASTRA. Cases with low and high triangularities are considered.

\section{Peeling-ballooning stability analysis}

Plasmas with high triangularity, $\delta=0.6$, and low triangularity, $\delta=0.2$, are considered. Other plasma parameters are held fixed in the reference cases: the minor radius $a=0.63 \mathrm{~m}$; major radius $R=1.69 \mathrm{~m}$; toroidal magnetic field $B_{T}=2.0 \mathrm{~T}$; plasma current $I=1.54 \mathrm{MA}$; elongation $\kappa=1.78$; central plasma density $n_{e}(0)=4.7 \times 10^{19} \mathrm{~m}^{-3}$; and central ion end electron temperatures $T_{e, i}=4 \mathrm{kEV}$. The TOQ equilibrium code [4] is used to generate a set of equilibria that covers the range of transport simulations for the plasma parameters given above. As long as the plasma geometry, toroidal magnetic field, and total plasma current are fixed in the transport simulations, ELMs are controlled by only the pressure gradient and bootstrap current. The shape of the electron density profile is kept unchanged in all scans; the electron density at the top of the pedestal is set to satisfy the dependence $n_{\text {ped }}=0.71\left\langle n_{e}\right\rangle$, which is observed in experiments. In the density scan, the entire density profile is scaled and in the temperature scan, the central temperature is kept fixed, while the pedestal temperature is changed. Both the bootstrap current and pressure gradient are changed in the density and temperature scans. The density scan provides more control of the bootstrap current, while the temperature scan provides more control of the normalized pressure gradient, $\alpha$, which is defined in this study as $\alpha=-\left(\mu_{0} / 2 \pi^{2}\right)(\partial p / \partial \psi)(\partial V / \partial \psi)\left(V / 2 \pi^{2} R\right)^{1 / 2}$, where $V$ is the plasma volume and $\psi$ is the poloidal flux. These equilibria are used in the BALOO, DCON, and ELITE codes to validate the peeling-ballooning stability criteria in the limits of different toroidal mode numbers. The BALOO code [4] is an infinite mode number ballooning stability code developed at General Atomics. The ideal MHD DCON code [5] is suitable for the stability analysis of low toroidal number ballooning and peeling modes, and the ELITE code [6] works well for the analysis of intermediate and high mode numbers. Since these codes are complementary, they can be used together to compute the stability criteria.

The results of the stability analysis are shown in Fig. 1. The high triangularity discharge has a larger stable region than the low triangularity discharge, which is consistent with experimental results and other MHD stability analysis [7]. In particular, the higher triangularity discharges have a larger second stability region, which is also consistent with the conclusion that higher triangularity discharges can more easily access the second ballooning stability region of parameter space [8]. 

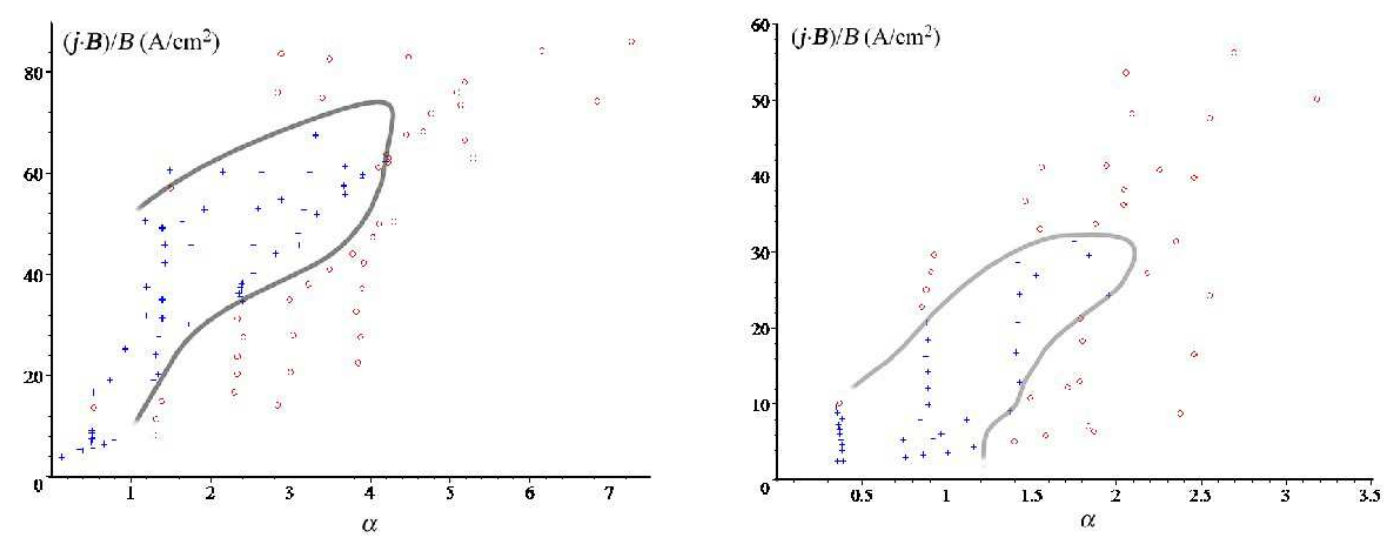

Fig. 1. ELM stability diagrams for discharges with (a) high $(\delta=0.6)$ and (b) low $(\delta=0.2)$ triangularity. Solid curve separates stable and unstable regions. The ' + ' symbol on the diagrams corresponds to the cases that are tested with the MHD stability codes appear to be stable; the 'o' symbol on the diagram correspond to the cases that appear to be unstable.

The peeling-ballooning threshold shown in Fig. 1 is parameterized using fifth order polynomials, which are implemented in the ASTRA transport code and used as the criteria to trigger ELM crashes in the transport simulations.

\section{Results of integrated transport simulations}

A reference scenario for ASTRA simulations is based on typical DIII-D geometry, using the parameters given at the beginning of previous Section. In addition, the electron, ion, and impurity density profiles, toroidal rotation velocity, $Z_{\text {eff }}$, the current density driven by the neutral beam injection (NBI) heating, and the auxiliary heating power deposited to electrons and ions, which are obtained from an analysis simulation of experimental data, are prescribed and fixed in form. For both the lower and higher triangularity discharges, the auxiliary heating power is varied from 3.5 MW to 7.0 MW in a series of simulations. The ELM frequencies as a function of auxiliary heating power for discharges with higher and lower triangularities are shown in Fig. 2. It can be seen that the ELM frequency increases with the heating power in the simulations, which is consistent with experimental observations in H-mode plasmas with type I ELMs. The change of the slope of the ELM frequency as a function of heating power, shown in Fig. 2, can be explained by the different scenarios that are followed for low and high auxiliary heating discharges. In the ASTRA simulations, the discharges with low auxiliary heating (below $7 \mathrm{MW}$ ) have ELM crashes that are triggered by a ballooning instability in the second stability limit, while the discharges with high heating power (above $7 \mathrm{MW}$ ) are triggered by a ballooning instability in the first stability limit.

\section{Summary}

An improved model is introduced for H-mode pedestal and ELMs [3]. A parameterized peelingballooning stability criterion is implemented in the model, based on detailed MHD analyses with the BALOO, DCON, and ELITE codes. Two different scenarios for ELM crashes in DIII-D discharges are shown. For the scenario with lower auxiliary heating power, ELMs are mostly caused by the ballooning instability in the second stability limit. For the scenario with higher auxiliary heating power (above $7 \mathrm{MW}$ ), ELMs might be caused by the ballooning instability in the first sta- 


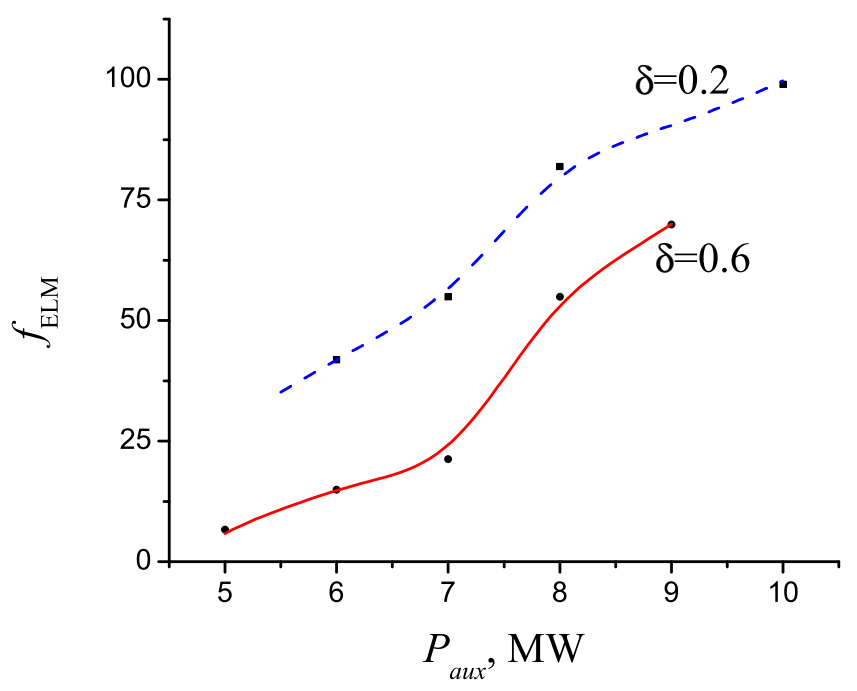

Fig. 2. The frequency of ELM crashes as function of the auxiliary heating power for discharges with low $(\delta=0.2)$ and high $(\delta=0.6)$ triangularities.

bility limit. Such ELM crashes are much less violent and more frequent. In general, the frequency of ELMs increases with the auxiliary heating power (as shown in Fig. 2), which is consistent with the experimental observations. The frequency of ELMs also depends on the plasma shaping. In particular, the dependence on the triangularity is studied in this paper. It is found that higher triangularity discharges have a larger stability region than lower triangularity discharges (compare Figs. 1 (a) and (b)). This observation is consistent with other MHD stability analysis [7, 8]. As result, ELMs in lower triangularity discharges are much more frequent than ELMs in higher triangularity discharges (as shown in Fig. 2).

In conclusion, it is clear that additional MHD stability studies are required. In this paper, ideal MHD stability codes are used, while resistivity and two-fluid effects are expected to be important. A preliminary study with the resistive MHD NIMROD [9] code is under way. In order to verify the results obtained with the MHD ideal stability code, a robust vacuum code should be used together with the NIMROD code which will be done in future studies.

\section{References}

[1] G. Pacher, H. Pacher, G. Janeschitz, et al., Plasma Phys. Control. Fusion 46, A257 (2004).

[2] J.-S. Lönnroth, V. V. Parail, A. Dnestrovskij, et al., Plasma Phys. Control. Fusion 46, 1197 (2004).

[3] A. Y. Pankin, I. Voitsekhovitch, G. Bateman, et al., Plasma Phys. Control. Fusion (2004).

[4] R. L. Miller and J. W. VanDam, Nucl. Fusion 28, 2101 (1987).

[5] A. H. Glasser and M. S. Chance, Bull. Am. Phys. Soc. 42, 1848 (1997).

[6] H. R. Wilson, P. B. Snyder, R. L. Miller, and G. T. A. 'Huysmans, Phys. Plasmas 9 (2002).

[7] P. B. Snyder, H. R. Wilson, et al., Nucl. Fusion 44, 320 (2004).

[8] T. Onjun, A. H. Kritz, G. Bateman, et al., Phys. Plasmas 11, 3006 (2004).

[9] C. R. Sovinec, D. C. Barnes, T. A. Gianakon, et al., Journal Comp. Physics (2004). 


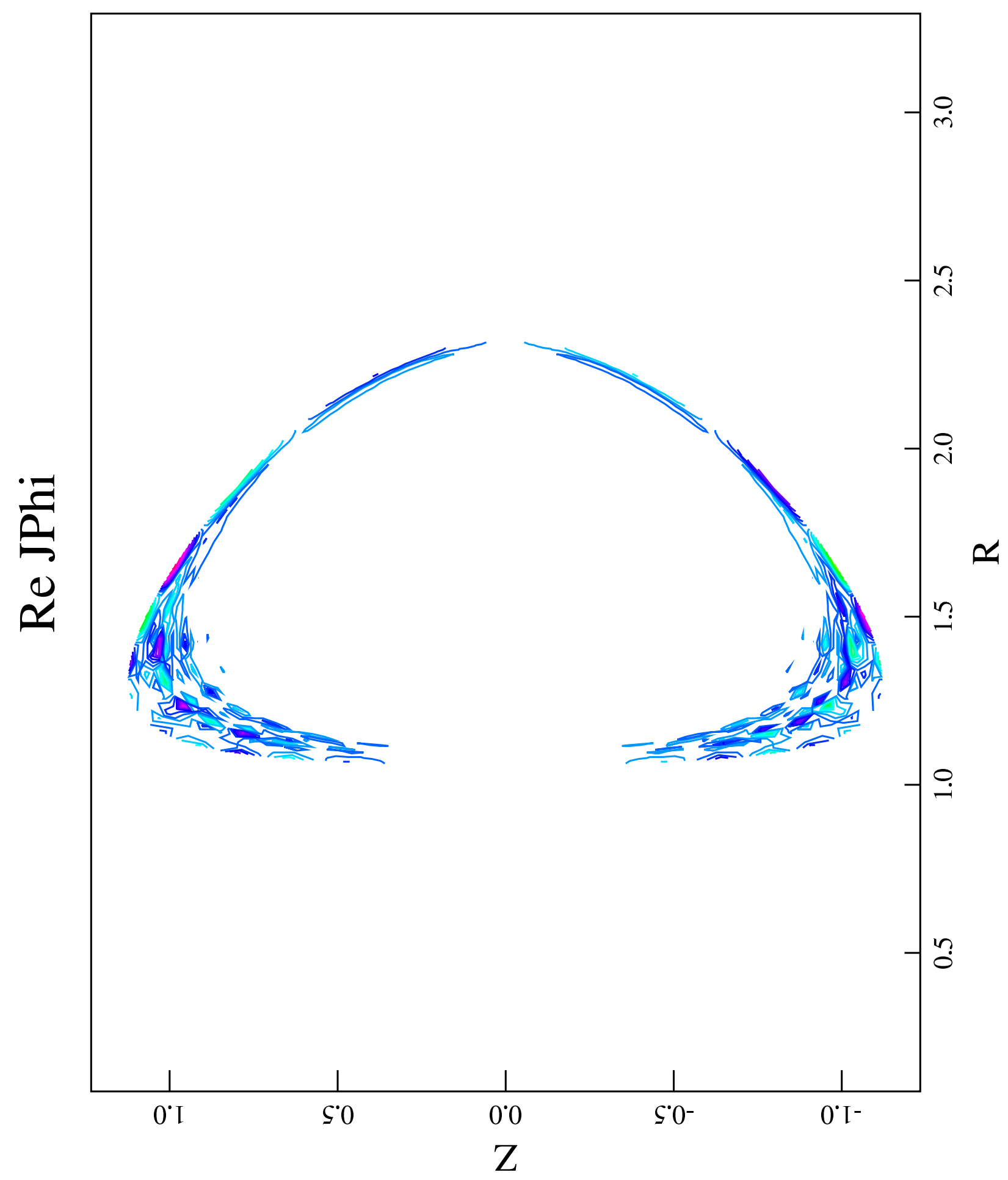




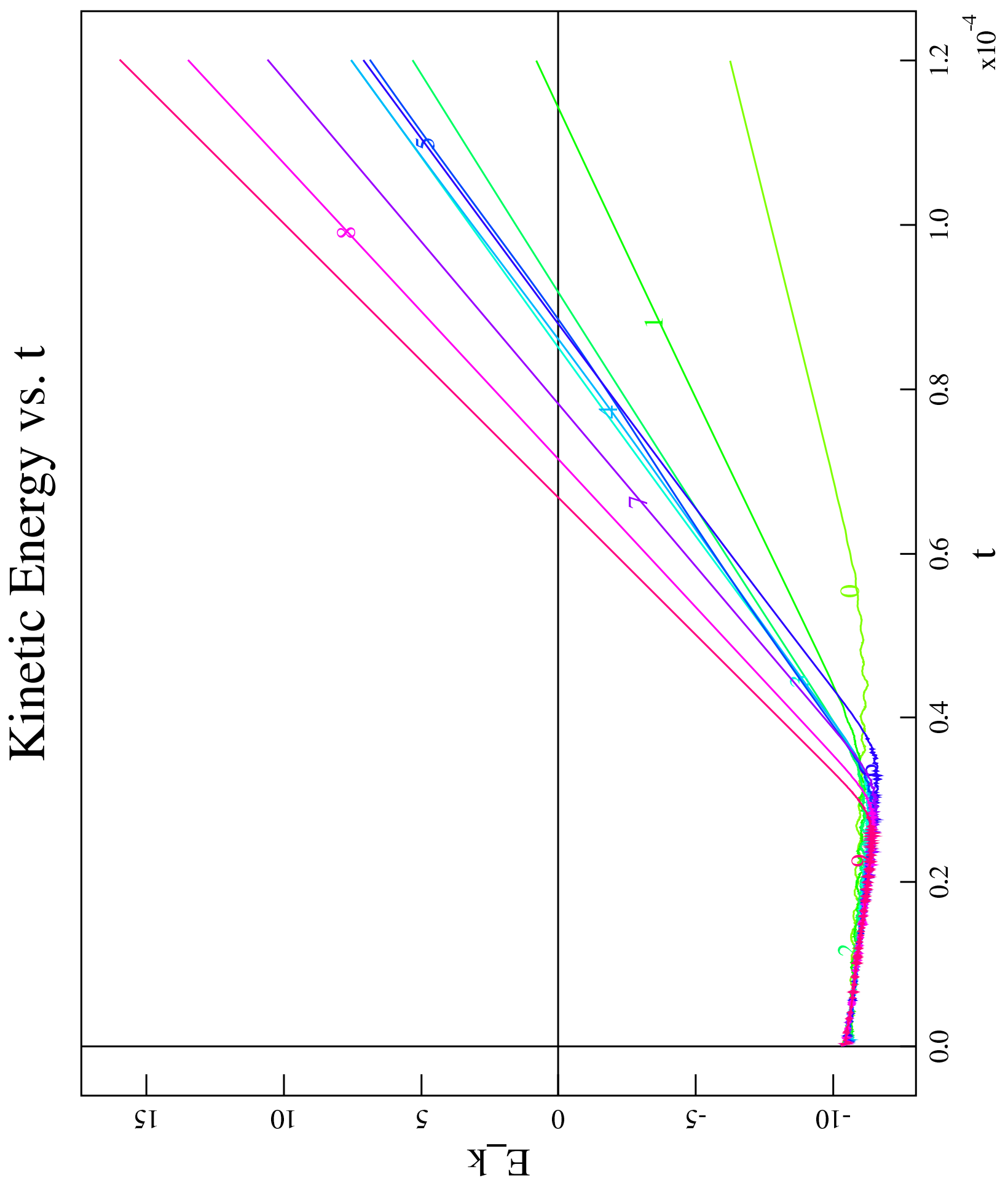




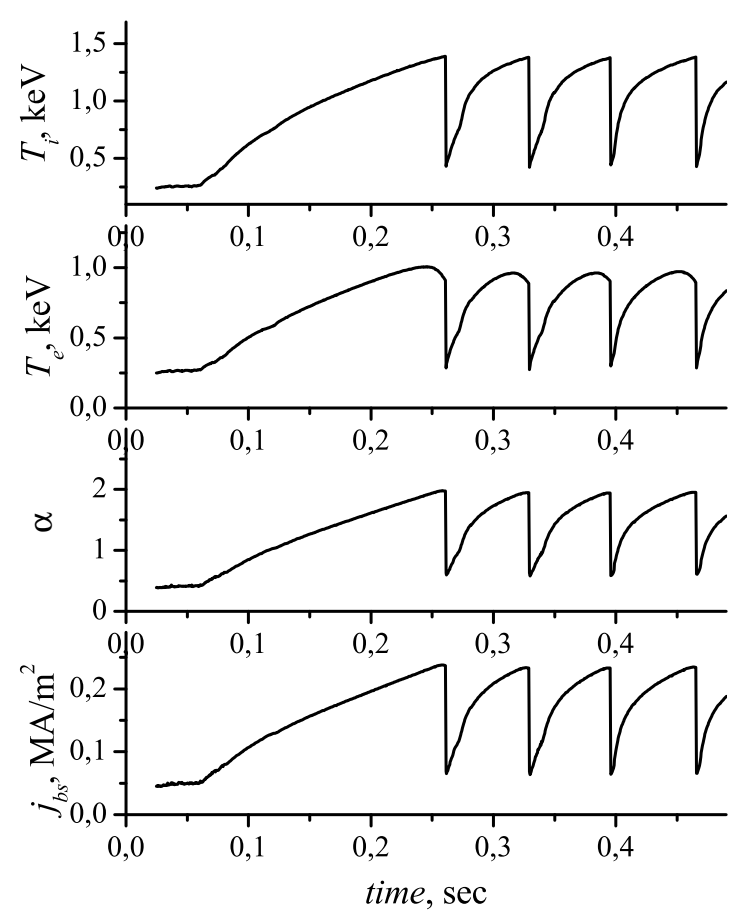




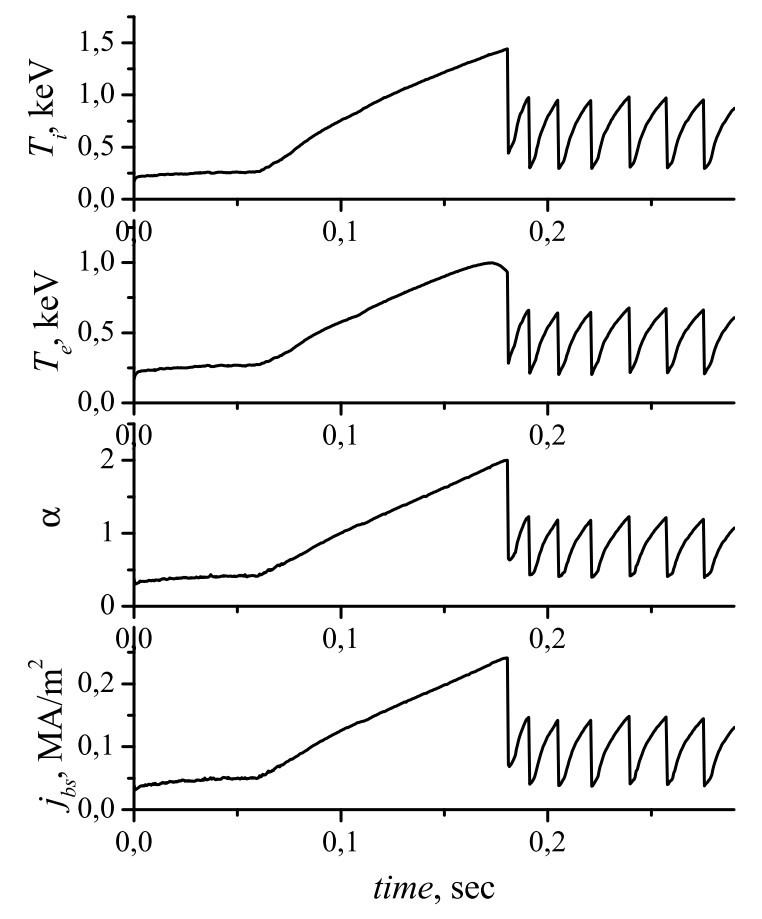




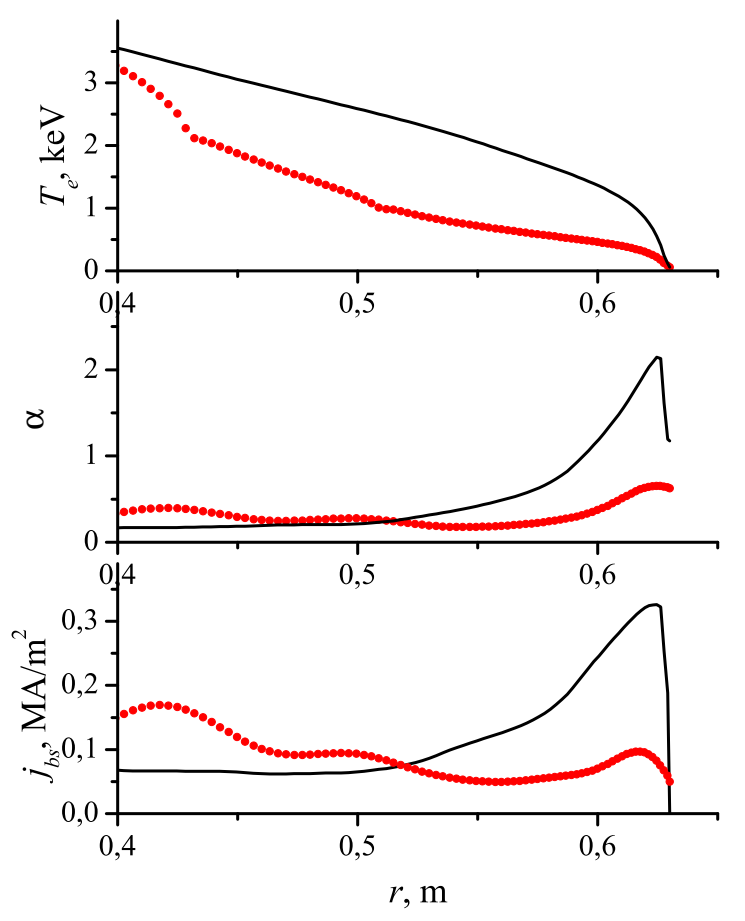

